\title{
A Novel MAGDM Approach With Proportional Hesitant Fuzzy Sets
}

\author{
Sheng-Hua Xiong ${ }^{1}$, Zhen-Song Chen ${ }^{2}{ }^{*}$, Kwai-Sang Chin ${ }^{3}$ \\ ${ }^{1}$ College of Civil Aviation Safety Engineering, Civil Aviation Flight University of China, \\ 46\# Section 4, Nanchang Road, \\ Guanghan, Sichuan 618307, People's Republic of China \\ E-mail:xsh@my.swjtu.edu.cn \\ ${ }^{2}$ School of Civil Engineering, Wuhan University, \\ Wuhan 430072, China \\ E-mail: zschen@whu.edu.cn \\ ${ }^{3}$ Department of Systems Engineering and Engineering Management, City University of Hong Kong, \\ Kowloon Tong, Hong Kong, People's Republic of China \\ E-mail: mekschin@cityu.edu.hk
}

Received 11 September 2017

Accepted 30 October 2017

\begin{abstract}
In this paper, we propose an extension of hesitant fuzzy sets, i.e., proportional hesitant fuzzy sets (PHFSs), with the purpose of accommodating proportional hesitant fuzzy environments. The components of PHFSs, which are referred to as proportional hesitant fuzzy elements (PHFEs), contain two aspects of information provided by a decision-making team: the possible membership degrees in the hesitant fuzzy elements and their associated proportions. Based on the PHFSs, we provide a novel approach to addressing fuzzy multi-attribute group decision making (MAGDM) problems. Different from the traditional approach, this paper first converts fuzzy MAGDM (expressed by classical fuzzy numbers) into proportional hesitant fuzzy multi-attribute decision making (represented by PHFEs), and then solves the latter through the proposal of a proportional hesitant fuzzy TOPSIS approach. In this process, preferences of the decision-making team are calculated as the proportions of the associated membership degrees. Finally, a numerical example and a comparison are provided to illustrate the reliability and effectiveness of the proposed approach.
\end{abstract}

Keywords: Fuzzy sets, hesitant fuzzy sets, proportional hesitant fuzzy sets, multi-attribute group decision making.

\section{Introduction}

The hesitant fuzzy problems are common in daily life, which have been initially interpreted by Torra ${ }^{1}$ as: "When defining the membership of an element, the difficulty of establishing the membership degree is not because we have a margin of error (as in A-
$I F S^{2}$ ), or some possibility distribution (as in type 2 fuzzy sets ${ }^{3}$ ) on the possible values, but because we have a set of possible values". To cope with these uncertainties produced by human being's hesitations, Torra and Narukawa ${ }^{1,5}$ expanded Zadeh's fuzzy sets (FSs) ${ }^{6}$ to another form of fuzzy multisets $^{7,8}$ : hesitant fuzzy sets (HFSs). It is worth noting

\footnotetext{
* Corresponding author
} 
that the HFSs can be applied to describe and handle the following two decision-making cases:

Case 1. Decision is made by one hesitant decision maker, who thinks the membership degree of an object belonging to a concept may have a set of possible values. For example, people's taste for dessert may change with mood. Good mood may taste more, whereas bad mood may taste less. Therefore, these different tastes constitute a HFS.

Case 2. Decision is made by a team, which contains no less than two decision makers. In this case, the team is automatically divided into more than one group according to the evaluation values of all decision makers: Different groups have distinct opinions on the membership degree, and each group cannot convince each other. For instance, different people may have diverse tastes for dessert, which similarly compose a HFS.

In the aforementioned two cases, the membership degree of an element to a set consists of several possible values in the real unit interval $[0,1]$. Case 1 derives from the dimension of "time", whereas "space" dimension is the main factor to promote the second case. Since the introduction of HFSs by Torra and Narukawa ${ }^{1,5}$, the state-of-the-art regarding HFSs mainly focuses on the following three aspects: aggregation operators, information measures and extensions.

The existing literature on aggregation operators is extensive. Following the intuitionistic fuzzy sets ${ }^{2}$, $\mathrm{Xia}$ and $\mathrm{Xu}^{9}$ defined the hesitant fuzzy weighted averaging operator, hesitant fuzzy weighted geometric operator, and many others. Similarly, a large number of other hesitant fuzzy aggregation operators were defined based on some basic aggregation operators, such as the hesitant fuzzy quasi-arithmetic aggregation operator ${ }^{11}$, hesitant fuzzy power geometric operators ${ }^{10}$, induced hesitant fuzzy aggregation operators ${ }^{11}$ and hesitant fuzzy geometric Bonferroni means ${ }^{12}$. Especially, in order to alleviate the computational complexity, several improved aggregation principles were also proposed regarding $\operatorname{HFSs}^{13,14,15}$.

The studies of hesitant fuzzy information measures are highly diversified, for instance, the distance and similarity measures on HFSs ${ }^{16,17}$, corre- lation coefficients over HFSs ${ }^{18,19}$ and entropy and cross entropy measures of HFSs ${ }^{20,21}$. Particularly, Farhadinia $^{21}$ explored the relationship among them and pointed that the distance, similarity and entropy measures are interchangeable under certain conditions. Furthermore, many extensions on HFSs (for example, the hesitant fuzzy linguistic terms sets ${ }^{22,23}$, interval-valued hesitant fuzzy sets ${ }^{21,24}$, higher order hesitant fuzzy sets ${ }^{17}$ and dual hesitant fuzzy sets ${ }^{25}$ ) have also been proposed with the purpose of modeling the hesitant fuzzy problem from various perspectives. Due to the fact that "the hesitant fuzzy set provides a more accurate representation of peoples hesitancy in stating their preferences over objects than the fuzzy set or its classical extensions" ${ }^{10}$, it has been widely and successfully applied to different practical areas, such as clustering analysis ${ }^{18,26}$, decision making ${ }^{19,22}$, and many others.

However, HFSs, including their extensions as mentioned above, are not applicable to addressing the case that a team could not reach agreement on a fuzzy decision (see Case 2), and the proportions of the associated membership degrees are measurable. For example, supposing a decision-making team consisted of ten members is invited to evaluate the membership degree of element $x \in X$ to set $E$, the evaluation result is as follows: one member (Group $A_{1}$ ) thinks the membership degree is 0.9; one member (Group $A_{2}$ ) thinks the membership degree is 0.7; two members (Group $A_{3}$ ) think the membership degree is 0.5 ; two members (Group $A_{4}$ ) think the membership degree is 0.3 ; and the rest four members (Group $A_{5}$ ) think the membership degree is 0.1 . Additionally, each group cannot convince each other. In this example, different groups hold diverse opinions on the degree of element $x \in X$ to set $E$ and their associated proportions are measurable. Utilizing the hesitant fuzzy element (HFE) ${ }^{9}$, this hesitant fuzzy problem can be expressed as $\{0.9,0.7,0.5,0.3,0.1\}$. However, the repeated rating values, such as four members think the membership degree is 0.1 in this example, are removed ${ }^{4}$. As mentioned by Peng et al. ${ }^{27}$, this removal is usually unreasonable, because values that appear just once may be more hesitant than a value repeated. Moreover, ignoring these repeated values may also loss part of preference infor- 
mation provided by the decision-making team.

Motivated by the aforementioned problem that may be faced in practice, this paper introduces the proportional hesitant fuzzy sets (PHFSs), which contain two aspects of information: the possible membership degrees in the hesitant fuzzy elements and their associated proportions. Utilizing proportional hesitant fuzzy elements $(\mathrm{PH}-$ FEs), the hesitant fuzzy problem mentioned in the last paragraph can be reasonably denoted as $\{(0.9,0.1),(0.7,0.1),(0.5,0.2),(0.3,0.2),(0.1,0.4)\}$, where $(0.1,0.4)$ represents Group $A_{5}$ thinks the membership degree is 0.1 and the proportion of Group $A_{5}$ is 0.4 . In PHFE, a large proportion indicates the decision-making team has a high preference for the associated membership degree, while the meaning for a small one is just converse. HFS therefore is a special case of PHFS, in which all membership degrees are regarded as sharing the same proportion. This novel extension, which meets the Fundamental Principle of a Generalization introduced by Rodríguez et al. ${ }^{4}$, provides a more accurate representation of people's hesitancy in stating their preferences over objects than HFS or its classical extensions.

Another motivation of this paper is to propose a novel approach for fuzzy multi-attribute group decision making (MAGDM), with the purpose of reasonably accommodating the information of human being's hesitations. The novel proposal first converts the fuzzy MAGDM into proportional hesitant fuzzy multi-attribute decision making (MADM) by calculating the proportions of the associated evaluation values, and then solves the MADM by using the proportional hesitant fuzzy TOPSIS ${ }^{36,37,38}$ approach. The key differences between the traditional approach and the novel approach proposed in this paper are as follows:

(1) Both the traditional and novel approaches first transform MAGDM into MADM. The difference is that this process in the former depends on the aggregation operator and evaluation information of all decision-makers, whereas that in the novel approach is only related to the evaluation information.

(2) The assessment information in both MAGDM and MADM is always represented by classical fuzzy numbers ${ }^{6}$ (FNs) in the traditional approach (see Stage 1 in Section 4). For the novel approach, it is expressed by FNs in MAGDM but PHFEs in the MADM.

(3) The novel approach can naturally reflect the preference information of the decision-making team.

The remaining sections of this paper are set up as follows: Section 2 briefly reviews several basic concepts related to this paper. Section 3 presents the concept of PHFSs, defines their basic operations and investigates a few of their properties. In Subsection 3.1, the distance measure on PHFSs is defined according to HFSs. Subsection 3.2 proposes an outranking method for the PHFEs. A novel fuzzy MAGDM approach based on the proportional hesitant fuzzy TOPSIS is proposed in Section 4. Especially, a numerical example about the performance evaluation of smart-phone is given to verify the developed approach and to demonstrate its practicality and effectiveness. In Section 5, a comparison with the hesitant fuzzy TOPSIS approach is provided to highlight the necessity of our conceptual extension in this paper. Sections 3 and 4 contain the main original contributions of this study. Section 6 concludes this paper.

\section{Preliminaries}

Torra and Narukawa ${ }^{1,5}$ originally proposed the concept of HFSs to deal with the situations where human beings have hesitancy in providing their preferences over objects in a decision-making process.

Definition 1. ${ }^{1,5}$ Let $X$ be a reference set, a hesitant fuzzy set (HFS) on $X$ is in terms of a function that when applied to $X$ returns a subset of $[0,1]$.

The HFS can be mathematically expressed as: ${ }^{9,16}$

$$
E=\left\{<x, h_{E}(x)>\mid x \in X\right\},
$$

where $h_{E}(x)$ is a set of values in $[0,1]$ that denotes the possible membership degrees of the element $x \in$ $X$ to the set $E$. For convenience, $\mathrm{Xia}$ and $\mathrm{Xu}^{9}$ called $h=h_{E}(x)$ as a hesitant fuzzy element (HFE).

For HFEs, Torra and Narukawa ${ }^{1,5}$ defined the following operations: 
Definition 2. ${ }^{1,5}$ Let $h, h_{1}$ and $h_{2}$ be three HFEs on the reference set $X$, then

(1) $h^{c}=\cup_{\gamma \in h}\{1-\gamma\}$;

(2) $h_{1} \cup h_{2}=\cup_{\gamma_{1} \in h_{1}, \gamma_{2} \in h_{2}}\left\{\gamma_{1} \vee \gamma_{2}\right\}$;

(3) $h_{1} \cap h_{2}=\cup_{\gamma_{1} \in h_{1}, \gamma_{2} \in h_{2}}\left\{\gamma_{1} \wedge \gamma_{2}\right\}$.

Definition 3. ${ }^{9}$ Let $h$ be a HFE on the reference set $X$, the score function of $h$ is defined as follows:

$$
s_{\mathrm{HFE}}(h)=\frac{\sum_{\gamma \in h} \gamma}{l(h)},
$$

where $l(h)$ is the number of values in $h$.

It is worth noting that score function $s_{\mathrm{HFE}}(h)$ is an arithmetic mean of values in HFE $h^{39}$, which represents its average assessment information. Some other forms of score functions for the HFE were similarly defined by Farhadinia ${ }^{40,41}$.

Definition 4. ${ }^{9}$ Let $h_{1}$ and $h_{2}$ be two HFEs on the reference set $X$,

(1) if $s_{\mathrm{HFE}}\left(h_{1}\right)>s_{\mathrm{HFE}}\left(h_{2}\right)$, then $h_{1}>h_{2}$;

(2) if $s_{\mathrm{HFE}}\left(h_{1}\right)=s_{\mathrm{HFE}}\left(h_{2}\right)$, then $h_{1}=h_{2}$.

Given two HFSs $A$ and $B$ on the reference set $X$, in most case, $l\left(h_{A}\left(x_{i}\right)\right) \neq l\left(h_{B}\left(x_{i}\right)\right)$ for $\forall x_{i} \in X$. Therefore, the shorter one should be extended with the corresponding optimistically/pessimistically larger value until both of them have the same length ${ }^{4,16}$. According to it, $\mathrm{Xu}$ and $\mathrm{Xia}^{16}$ defined the hesitant normalized Hamming distance.

Definition 5. ${ }^{16}$ Let $A$ and $B$ be two HFSs on the reference set $X=\left\{x_{1}, x_{2}, \ldots, x_{n}\right\}$, then the hesitant normalized Hamming distance is

$$
d_{\mathrm{HFS}}(A, B)=\frac{1}{n} \sum_{i=1}^{n}\left[\frac{1}{l_{x_{i}}} \sum_{j=1}^{l_{x_{i}}}\left|h_{A}^{\sigma(j)}\left(x_{i}\right)-h_{B}^{\sigma(j)}\left(x_{i}\right)\right|\right],
$$

where $l_{x_{i}}=\max \left\{l\left(h_{A}\left(x_{i}\right)\right), l\left(h_{B}\left(x_{i}\right)\right)\right\}$, and $h_{A}^{\sigma(j)}\left(x_{i}\right)$ and $h_{B}^{\sigma(j)}\left(x_{i}\right)$ are the $j$ th largest values in $h_{A}\left(x_{i}\right)$ and $h_{B}\left(x_{i}\right)$, respectively.

\section{Proportional hesitant fuzzy sets}

HFSs provide us a useful tool to describe and address another form of fuzzy problem derived from human being's hesitation. However, as mentioned in Section 1, they cannot reasonably handle Case 2 with the proportions of the membership degrees are measurable. To cope with it, in this section, the concept of the proportional hesitant fuzzy sets and some properties regarding them are introduced on the basis of HFSs.

Definition 6. Let $X$ be a reference set, the proportional hesitant fuzzy set (PHFS) $E$ on $X$ is represented by the following mathematical notation:

$E=\left\{\left\langle x, p h_{E}(x)\right\rangle \mid x \in X\right\}=\left\{\left\langle x,\left(h_{E}(x), p_{E}(x)\right)\right\rangle \mid x \in X\right\}$, where

(a) $h_{E}(x)=\left\{\gamma_{1}, \gamma_{2}, \cdots, \gamma_{n}\right\}$ is a set of values in $[0,1]$, which represents $n$ kinds of possible membership degrees of the element $x$ to set $E$; and

(b) $p_{E}(x)=\left\{\tau_{1}, \tau_{2}, \cdots, \tau_{n}\right\}$ is a set of values in $[0,1]$, where $\tau_{i}(i=1,2, \cdots, n)$ denotes the proportion of membership degree $\gamma_{i}(i=1,2, \cdots, n)$ and $\sum_{i=1}^{n} \tau_{i}=1$.

For convenience, we call $p h=p h_{E}(x)$ as a proportional hesitant fuzzy element (PHFE).

The PHFS is a three-dimensional fuzzy set, which can clearly and carefully show us the hesitant assessment information provided by decisionmaking team on both the multiple membership degrees and their associated proportions. HFS therefore is a special case of PHFS, in which all membership degrees share the same proportion.

Proportional information, to our knowledge, has been originally considered into the fuzzy (linguistic term ${ }^{28,29}$ ) sets by Wang and $\mathrm{Hao}^{30}$, who represented the linguistic information by proportional 2-tuples. As a natural generalization of the Wang and Hao model, Zhang et al..$^{31}$ proposed the distribution assessment in a linguistic term set, in which symbolic proportions are assigned to all linguistic terms. Zhang et al. illustrated their model with an example that a football coach used the terms in $S=\left\{s_{-2}=\right.$ very poor,$s_{-1}=$ poor,$s_{0}=$ average, $s_{1}=$ good, $s_{2}=$ very good $\}$ to evaluate a player's level. For the ten games he was involved 
in, three times were judged as $s_{-1}$, two times were judged as $s_{1}$, and the other five times were judged as $s_{2}$. Then, the evaluations of the coach can be described as the linguistic distribution assessment $\left\{\left(s_{-2}, 0\right),\left(s_{-1}, 0.3\right),\left(s_{0}, 0\right),\left(s_{1}, 0.2\right),\left(s_{2}, 0.5\right)\right\}$. Wu and $\mathrm{Xu}^{32}$ focused on a special situation, where the possible linguistic terms provided by the decision maker are assigned with the same proportion. Inspired by pioneer works, more and more attention has been paid to the linguistic distribution assessment ${ }^{33,34,35}$. Although the PHFSs are similarly defined to handle the proportional uncertainty problem, they are quite different from these studies!

(1) The research objects in these studies are the linguistic information, whereas it is the hesitant fuzzy information for the PHFSs.

(2) According to Zhang et al.'s example, these studies can be used to cope with the proportional hesitant information deriving from the "time" dimension as shown in Case 1 of Section 1. The PHFSs are developed to model the proportional hesitant uncertainty resulting from the "space" dimension (see Case 2 in Section 1).

Note that $p h_{1} * p h_{2}=\left\{\left(\gamma_{1}, \tau_{1}\right),\left(\gamma_{1}, \tau_{2}\right),\left(\gamma_{2}, \tau_{3}\right)\right.$, $\left.\left(\gamma_{2}, \tau_{3}\right),\left(\gamma_{3}, \tau_{4}\right)\right\}$ should be expressed as $p h_{1} * p h_{2}=$ $\left\{\left(\gamma_{1}, \tau_{1}+\tau_{2}\right),\left(\gamma_{2}, 2 \tau_{3}\right),\left(\gamma_{3}, \tau_{4}\right)\right\}$ according to set theory, where " $*$ " is an operation between PHFEs.

Definition 7. Let $X$ be a reference set, for any $x \in X$, call

(1) $p h_{E}(x)=\{(0,1)\}$ as the empty proportional hesitant fuzzy set, denoted by $\emptyset$;

(2) $p h_{E}(x)=\{(1,1)\}$ as the full proportional hesitant fuzzy set, denoted by $\Omega$.

Definition 8. Given a PHFS represented by its PHFE $p h$, the complement of $p h$ is

$$
p h^{c}=\cup_{(\gamma, \tau) \in p h}\{(1-\gamma, \tau)\} .
$$

The complement of the PHFE is defined in an intuitive manner. According to the intuitionistic fuzzy sets, ${ }^{2}$ if the membership degree of an object belonging to a concept is $\gamma$, then $1-\gamma$ represents the non-membership and indeterminacy degrees of that object belonging to the same concept. ${ }^{42}$ Consequently, Definition 8 can be interpreted as these decision makers who think the membership degree of an object belonging to a concept is $\gamma$ may also hold the view that the non-membership and indeterminacy degrees of that object belonging to the same concept are $1-\gamma$.

Theorem 1. The complement is involutive, i.e.,

$$
\left(p h^{c}\right)^{c}=p h .
$$

Proof. Trivial as $1-(1-\gamma)=\gamma$ for any $(\gamma, \tau) \in$ $p h$. Consequently, $\left(p h^{c}\right)^{c}=p h$.

Let $p h_{1}$ and $p h_{2}$ be two PHFEs on the reference set $X$, and suppose the membership degree of the $x \in X$ to the set " 1 " and that to the set " 2 " are mutually independent. The following union and intersection operations on PHFEs are defined from the angle of probability.

Definition 9. Let $p h_{1}$ and $p h_{2}$ be two PHFEs on the reference set $X$, then

(1) $p h_{1} \cup p h_{2}=\cup_{\left(\gamma_{1}, \tau_{1}\right) \in p h_{1},\left(\gamma_{2}, \tau_{2}\right) \in p h_{2}}\left\{\left(\gamma_{1} \vee \gamma_{2}, \tau_{1} \tau_{2}\right)\right\}$;

(2) $p h_{1} \cap p h_{2}=\cup_{\left(\gamma_{1}, \tau_{1}\right) \in p h_{1},\left(\gamma_{2}, \tau_{2}\right) \in p h_{2}}\left\{\left(\gamma_{1} \wedge \gamma_{2}, \tau_{1} \tau_{2}\right)\right\}$.

Based on PHFSs, some relationships can be further established for these operations.

Theorem 2. Let A, B and $C$ be three PHFSs on the reference set $X$, then

(1) $A \cup \emptyset=A, A \cap \Omega=A, A \cap \emptyset=\emptyset, A \cup \Omega=\Omega$;

(2) $A \cup B=B \cup A, A \cap B=B \cap A$;

(3) $(A \cup B) \cup C=A \cup(B \cup C),(A \cap B) \cap C=A \cap$ $(B \cap C)$;

\footnotetext{
$\dagger$ This paper in part is inspired by the rapid development of semantics for evaluation information as we have briefly introduced here.

$\ddagger$ In fact, PHFSs can as well be utilized to characterize the proportional hesitant information derived from the "time" dimension, which can be generated from a dynamic evaluation process conducted by a single decision maker. The information representation construction in this paper, however, focuses on the manifestation of group evaluations, therefore, we place restrictions on our discussion to the management of proportional hesitant group decision making resulting from the "space" dimension. Application of PHFSs in the modelling of individual evaluations is not discussed at the current stage to keep the paper stay focused, and we would like to leave it for future investigation, mainly because this issue does not jeopardize methodological integrity or pose any theoretical barriers for comprehension.
} 
(4) $(A \cup B)^{c}=A^{c} \cap B^{c},(A \cap B)^{c}=A^{c} \cup B^{c}$.

Proof. Following Definition 7, (1) is easy to verify. (2) Since

$$
\begin{aligned}
p h_{A} \cup p h_{B} & =\underset{\left(\gamma_{A}, \tau_{A}\right) \in p h_{A},\left(\gamma_{B}, \tau_{B}\right) \in p h_{B}}{\cup}\left\{\left(\gamma_{A} \vee \gamma_{B}, \tau_{A} \tau_{B}\right)\right\} \\
& =\underset{\left(\gamma_{B}, \tau_{B}\right) \in p h_{B},\left(\gamma_{A}, \tau_{A}\right) \in p h_{A}}{\cup}\left\{\left(\gamma_{B} \vee \gamma_{A}, \tau_{B} \tau_{A}\right)\right\} \\
& =p h_{B} \cup p h_{A},
\end{aligned}
$$

then, $A \cup B=B \cup A$. Similarly, $A \cap B=B \cap A$.

(3) Since

$$
\begin{aligned}
& \left(p h_{A} \cup p h_{B}\right) \cup p h_{C} \\
& =\underset{\left(\gamma_{A}, \tau_{A}\right) \in p h_{A},\left(\gamma_{B}, \tau_{B}\right) \in p h_{B}}{\cup}\left\{\left(\gamma_{A} \vee \gamma_{B}, \tau_{A} \tau_{B}\right)\right\} \cup p h_{C} \\
& =\underset{\left(\gamma_{A}, \tau_{A}\right) \in p h_{A},\left(\gamma_{B}, \tau_{B}\right) \in p h_{B},\left(\gamma_{C}, \tau_{C}\right) \in p h_{C}}{\cup}\left\{\left(\left(\gamma_{A} \vee \gamma_{B}\right) \vee \gamma_{C},\right.\right. \\
& \left.\left.\left(\tau_{A} \tau_{B}\right) \tau_{C}\right)\right\} \\
& =\underset{\left(\gamma_{A}, \tau_{A}\right) \in p h_{A},\left(\gamma_{B}, \tau_{B}\right) \in p h_{B},\left(\gamma_{C}, \tau_{C}\right) \in p h_{C}}{\cup}\left\{\left(\gamma_{A} \vee\left(\gamma_{B} \vee \gamma_{C}\right),\right.\right. \\
& \left.\left.\tau_{A}\left(\tau_{B} \tau_{C}\right)\right)\right\} \\
& =p h_{A} \cup \underset{\left(\gamma_{B}, \tau_{B}\right) \in p h_{B},\left(\gamma_{C}, \tau_{C}\right) \in p h_{C}}{\cup}\left\{\left(\gamma_{B} \vee \gamma_{C}, \tau_{B} \tau_{C}\right)\right\} n \\
& =p h_{A} \cup\left(p h_{B} \cup p h_{C}\right) \text {, }
\end{aligned}
$$

then, $(A \cup B) \cup C=A \cup(B \cup C)$. Similarly, $(A \cap B) \cap$ $C=A \cap(B \cap C)$.

(4) Since

$$
\begin{aligned}
& \left(p h_{A} \cup p h_{B}\right)^{c} \\
& =\underset{\left(\gamma_{A}, \tau_{A}\right) \in p h_{A},\left(\gamma_{B}, \tau_{B}\right) \in p h_{B}}{\cup}\left\{\left(1-\gamma_{A} \vee \gamma_{B}, \tau_{A} \tau_{B}\right)\right\} \\
& =\underset{\left(\gamma_{A}, \tau_{A}\right) \in p h_{A},\left(\gamma_{B}, \tau_{B}\right) \in p h_{B}}{\cup}\left\{\left(\left(1-\gamma_{A}\right) \wedge\left(1-\gamma_{B}\right), \tau_{A} \tau_{B}\right)\right\} \\
& =\left(p h_{A}\right)^{c} \cap\left(p h_{B}\right)^{c},
\end{aligned}
$$

then, $(A \cup B)^{c}=A^{c} \cap B^{c}$. Similarly, $(A \cap B)^{c}=A^{c} \cup$ $B^{c}$.

\section{Distance measure for PHFSs}

Distance measures are fundamentally important in various fields such as pattern recognition, market prediction, and decision making. According to the distance measure for HFSs ${ }^{16}$, the axioms of the distance measure for PHFSs are defined as follows.

Definition 10. Let $A$ and $B$ be two PHFSs on the reference set $X$, then the distance measure between them is defined as $d(A, B)$, which should meet the following properties:

(1) Boundary: $0 \leqslant d(A, B) \leqslant 1$;

(2) Reflexivity: $d(A, B)=0$ if and only if $A=B$;

(3) Symmetry: $d(A, B)=d(B, A)$.

Similar to HFSs, if $l(p h)$ represents the number of elements in PHFE $p h$, it is difficult to calculate the distance measure between PHFSs $A$ and $B$, because $l\left(p h_{A}(x)\right)$ is usually not equal to $l\left(p h_{B}(x)\right)$ for any $x \in X$. Supposing $l_{x}=\max \left\{l\left(p h_{A}(x)\right), l\left(p h_{B}(x)\right)\right\}$, as with related literature ${ }^{16}$, this problem can be handled by the following two steps:

(1) Ordering: arrange the elements in $p h_{A}(x)$ and $p h_{B}(x)$ in decreasing order according to the product values of the membership degrees and their associated proportions;

(2) Adding: add the PHFE whose $l(*)$ is smaller with element $(0,0)$ several times until both of them have the same number of elements, i.e., $l_{x}$.

In the real-life group decision-making context, both the evaluation opinions (membership degrees) and the preferences (proportions) of the decisionmaking team are important to the final decisionmaking result. Consequently, an element in the PHFE with the largest membership degree does not mean it must be ordered in the first place, but the one makes the greatest contribution (the largest product value) should be. It is noteworthy that the membership degree of the element added into each PHFE can be any value from 0 to 1 , because its associated proportion is always equal to 0 (otherwise, it is no longer a PHFE since the total proportion is more than 1).

Example 1. Let $p h_{A}=\{(0.1,0.4),(0.3,0.1),(0.6,0.2)$, $(0.7,0.1),(0.9,0.2)\}$ and $p h_{B}=\{(0.3,0.1),(0.5,0.7)$, $(0.7,0.2)\}$ be two PHFEs on the reference set $X . \quad$ It is clear that $l\left(p h_{A}\right)=5$ and $l\left(p h_{B}\right)=3$. Utilizing the ordering method, $p h_{A}^{\sigma}=\{(0.9,0.2),(0.6,0.2),(0.7,0.1),(0.1,0.4)$, $(0.3,0.1)\}$ and $p h_{B}^{\sigma}=\{(0.5,0.7),(0.7,0.2),(0.3,0.1)\}$. 
On the other hand, $p h_{B}$ should be added with $(0,0)$ twice because $l\left(p h_{A}\right)-l\left(p h_{B}\right)=2$ and then it becomes $p h_{B^{\prime}}^{\sigma}=\{(0.5,0.7),(0.7,0.2),(0.3,0.1)$, $(0,0),(0,0)\}$.

Distance calculation is a useful tool to measure the differences between two systems, therefore the distance measure for PHFSs should include the following two parts: opinion differences (i.e., the differences between membership degrees) and preference differences (i.e., the differences between proportions). Due to the fact that an increase in either part will result in an incremental distance, the proportional hesitant normalized Hamming distance then can be defined as follows.

Definition 11. Let $A$ and $B$ be two PHFSs on the reference set $X=\left\{x_{1}, x_{2}, \ldots, x_{n}\right\}$, then the proportional hesitant normalized Hamming distance is

$$
\begin{aligned}
& d(A, B)=\frac{1}{n} \sum_{i=1}^{n}\left[\frac{1}{2 l_{x_{i}}} \sum_{j=1}^{l_{x_{i}}} \mid \gamma_{A}^{\sigma(j)}\left(x_{i}\right) \cdot \tau_{A}^{\sigma(j)}\left(x_{i}\right)\right. \\
& \left.-\gamma_{B}^{\sigma(j)}\left(x_{i}\right) \cdot \tau_{B}^{\sigma(j)}\left(x_{i}\right)|+| \tau_{A}^{\sigma(j)}\left(x_{i}\right)-\tau_{B}^{\sigma(j)}\left(x_{i}\right) \mid\right],
\end{aligned}
$$

where $l_{x_{i}}=\max \left\{l\left(p h_{A}\left(x_{i}\right)\right), l\left(p h_{B}\left(x_{i}\right)\right)\right\}, \quad$ and $\gamma_{A}^{\sigma(j)}\left(x_{i}\right) \cdot \tau_{A}^{\sigma(j)}\left(x_{i}\right)$ and $\gamma_{B}^{\sigma(j)}\left(x_{i}\right) \cdot \tau_{B}^{\sigma(j)}\left(x_{i}\right)$ are the $j$ th largest product value in PHFEs $p h_{A}\left(x_{i}\right)$ and $p h_{B}\left(x_{i}\right)$, respectively.

The distance measure on PHFEs defined in Definition 11 has a lot of advantages. First, the internal elements for each PHFE are sequenced on the basis of their corresponding "contributions", which include the membership and proportion information of the decision-making system. Moreover, because the element added into the PHFE can be represented as the form of $(a, 0), a \in[0,1]$, any addition does not change the distance measure value between two $\mathrm{PH}$ FEs.

\section{A comparison method for proportional hesitant fuzzy elements}

Similar to the distance measure on PHFSs, the comparison method for PHFEs should take the membership and proportion information into account simultaneously. We first introduce the following two functions.
Definition 12. Let $p h$ be a PHFE on the reference set $X$, the score function of $p h$ is defined as

$$
s(p h)=\sum_{(\gamma, \tau) \in p h} \gamma \cdot \tau,
$$

and the deviation function of $p h$ is defined as

$$
t(p h)=\sum_{(\gamma, \tau) \in p h} \tau \cdot(\gamma-s(p h))^{2} .
$$

The score and deviation functions of the PHFE derive from the expectation and variance of random variables, respectively. Similarly, the score function represents the average assessment information contained in PHFE $p h$.

Combing with the distance measure, the comparison method for PHFEs can be defined as follows.

Definition 13. Let $p h_{1}$ and $p h_{2}$ be two PHFEs on the reference set $X$,

(1) if $s\left(p h_{1}\right)>s\left(p h_{2}\right)$, then $p h_{1}>p h_{2}$;

(2) if $s\left(p h_{1}\right)=s\left(p h_{2}\right)$ and $t\left(p h_{1}\right)<t\left(p h_{2}\right)$, then $p h_{1}>p h_{2}$

(3) if $s\left(p h_{1}\right)=s\left(p h_{2}\right), t\left(p h_{1}\right)=t\left(p h_{2}\right)$,

(a) and $d\left(\left\{p h_{1}\right\}, \Omega\right)=d\left(\left\{p h_{2}\right\}, \Omega\right)$, then $p h_{1}=p h_{2}$;

(b) and $d\left(\left\{p h_{1}\right\}, \Omega\right)<d\left(\left\{p h_{2}\right\}, \Omega\right)$, then $p h_{1}>p h_{2}$.

where $\Omega$ is the full proportional hesitant fuzzy set and $d(A, B)$ is the distance measure for PHFSs.

Formula (1) can be interpreted as the larger the average evaluation information, the larger the associated PHFE. If two PHFEs contain the same average evaluation information, formula (2) indicates the less the deviation of the evaluation values, the larger the associated PHFE. Furthermore, because the full proportional hesitant fuzzy set $\Omega$ represents the largest evaluation information, the closer to it, the larger the associated PHFE.

\section{A novel approach for fuzzy multiple attribute group decision making}

Formally, an MAGDM problem can be concisely described as $s(s \geqslant 2)$ decision makers $D M_{k}(k=$ $1,2, \ldots, s)$ provide their evaluation values over $m$ alternatives $A_{i}(i=1,2, \ldots, m)$ under $n$ attributes 
$C_{j}(j=1,2, \ldots, n)$ to find the best option from all of the feasible alternatives. For convenience, let $M=$ $\{1,2, \ldots, m\}, N=\{1,2, \ldots, n\}$ and $S=\{1,2, \ldots, s\}$. Suppose decision maker $D M_{k}$ use the classical FN to provide his evaluation value about alternative $A_{i}$ under attribute $C_{j}$, which is denoted as $\mu_{i j}^{k}(i \in M ; j \in$ $N ; k \in S)$. Then, $s$ fuzzy evaluation matrices $U_{k}=$ $\left[\mu_{i j}^{k}\right]_{m \times n}(k \in S)$ can be attained. In the traditional fuzzy MAGDM approach, the following two basic stages are usually utilized to solve this problem:

Stage 1: Transform the fuzzy MAGDM into the fuzzy MADM by using the fuzzy aggregation operator. Note that the evaluation information is always represented by the FNs in both the MAGDM and MADM;

Stage 2: Solve the fuzzy MADM problem.

Up to now, the alternative preferences of the decision-making team have received a growing number of attentions in the hesitant fuzzy group decision making. ${ }^{43,44,45}$ Due to the fact that different decision makers may be heterogeneous with respect to their tastes for diverse attributes and alternatives, the preferences of the decision-making team for each alternative under different attributes should similarly be considered. Especially, Dong et $\mathrm{al},{ }^{46}$ proposed a resolution framework for the complex and dynamic MAGDM problem, in which decision makers are supposed to have different interests and use heterogeneous individual sets of attributes to evaluate the individual alternatives. Dong et al, ${ }^{47}$ meaningfully considered the complex and dishonest context, where a decision maker can strategically set the preferences to obtain her/his desired ranking of alternatives. This paper from a different perspective takes into account heterogeneous individual preferences and converts them into the proportions of the associated membership degrees in each PHEs. The fuzzy MAGDM problem can be solved as follows: First, based on fuzzy evaluation matrices $U_{k}=\left[\mu_{i j}^{k}\right]_{m \times n}(k \in S)$, the overall evaluation matrix $U=\left[p h_{i j}\right]_{m \times n}$ can be attained by calculating the proportions of the associated membership degrees (see Example 2), i.e., the preferences of the decision-making team. Because the evaluation value $\mu_{i j}^{k}(i \in M ; j \in N ; k \in S)$ is given by the classical FN, then $p h_{i j}(i \in M ; j \in N)$ is a PHFE, which consists of several possible evaluation values of alternative $A_{i}$ under attribute $C_{j}$ and their associated proportions. After that, we only need to solve the fuzzy MADM problem under the proportional hesitant fuzzy environment.

Example 2. Suppose three HRs use FNs to evaluate two candidates under the communication skill $\left(C_{1}\right)$ and the learning skill $\left(C_{2}\right)$. The detailed evaluation values are $\mu_{11}^{1}=0.9, \mu_{12}^{1}=0.5, \mu_{11}^{2}=0.7$, $\mu_{12}^{2}=0.6, \mu_{11}^{3}=0.9, \mu_{12}^{3}=0.5$ for Candidate 1 , and $\mu_{21}^{1}=0.8, \mu_{22}^{1}=0.6, \mu_{21}^{2}=0.7, \mu_{22}^{2}=0.5$, $\mu_{21}^{3}=0.7, \mu_{22}^{3}=0.3$ for Candidate 2. Then, the fuzzy MAGDM is

$$
\begin{gathered}
U_{1}=\left[\begin{array}{ll}
0.9 & 0.5 \\
0.8 & 0.6
\end{array}\right], \quad U_{2}=\left[\begin{array}{ll}
0.7 & 0.6 \\
0.7 & 0.5
\end{array}\right], \\
U_{3}=\left[\begin{array}{ll}
0.9 & 0.5 \\
0.7 & 0.3
\end{array}\right] .
\end{gathered}
$$

For Candidate 1, the evaluation values under the communication skill are $0.9,0.7$ and 0.9 with respect to the three HRs. Therefore, the proportion of membership degree 0.9 is $2 / 3$ and that of membership degree 0.7 is $1 / 3$, then the overall evaluation value can be represented by PHFE $\{(0.9,2 / 3),(0.7,1 / 3)\}$. Similarly, the overall evaluation matrix is

$$
\begin{aligned}
& U= \\
& {\left[\begin{array}{lc}
\left\{\left(0.9, \frac{2}{3}\right),\left(0.7, \frac{1}{3}\right)\right\} & \left\{\left(0.6, \frac{1}{3}\right),\left(0.5, \frac{2}{3}\right)\right\} \\
\left\{\left(0.8, \frac{1}{3}\right),\left(0.7, \frac{2}{3}\right)\right\} & \left\{\left(0.6, \frac{1}{3}\right),\left(0.5, \frac{1}{3}\right),\left(0.3, \frac{1}{3}\right)\right\}
\end{array}\right],}
\end{aligned}
$$

which can be considered as a proportional hesitant fuzzy MADM problem.

Table 1 shows the comparisons of the novel and traditional approaches in the stage of transforming the MAGDM into the MADM. It is worth noting that the fuzzy MAGDM is converted into the proportional hesitant fuzzy MADM. In the fuzzy MAGDM, the evaluation values are expressed by the FNs, whereas they are the PHFEs in the proportional hesitant fuzzy MADM. The novel approach is therefore different from the traditional fuzzy MAGDM approach, in which the evaluation values are always represented by the FNs as shown in Stage 1 .

Example 2 also indicates that obtaining the proportional information does not require the decisionmaking team to provide extra evaluation information. If the proportional information is ignored, the 
Table 1. Comparisons of the novel and traditional approaches on transforming the MAGDM into the MADM

\begin{tabular}{|c|c|c|c|}
\hline & & Novel approach & Traditional apporach \\
\hline \multirow[t]{2}{*}{ Similarity } & $\begin{array}{l}\text { Representation of the initial evaluation } \\
\text { information (MAGDM) }\end{array}$ & FNs & FNs \\
\hline & $\begin{array}{l}\text { Representation of the overall evaluation } \\
\text { information (MADM) }\end{array}$ & PHFEs & FNs \\
\hline \multirow[t]{2}{*}{ Differences } & $\begin{array}{l}\text { Extra information required for transfor- } \\
\text { ming the MAGDM into the MADM }\end{array}$ & Nothing & $\begin{array}{l}\text { Aggregation } \\
\text { operator }\end{array}$ \\
\hline & $\begin{array}{l}\text { Can naturally reflect the preference inf- } \\
\text { ormation of the decision-making team }\end{array}$ & Yes & No \\
\hline
\end{tabular}

overall evaluation value for Candidate 1 under the communication skill then is $\{0.9,0.7\}$, in which the preferences of the decision-making team are ignored as well. Consequently, the novel approach can nat- urally consider that preferences into the decisionmaking process.

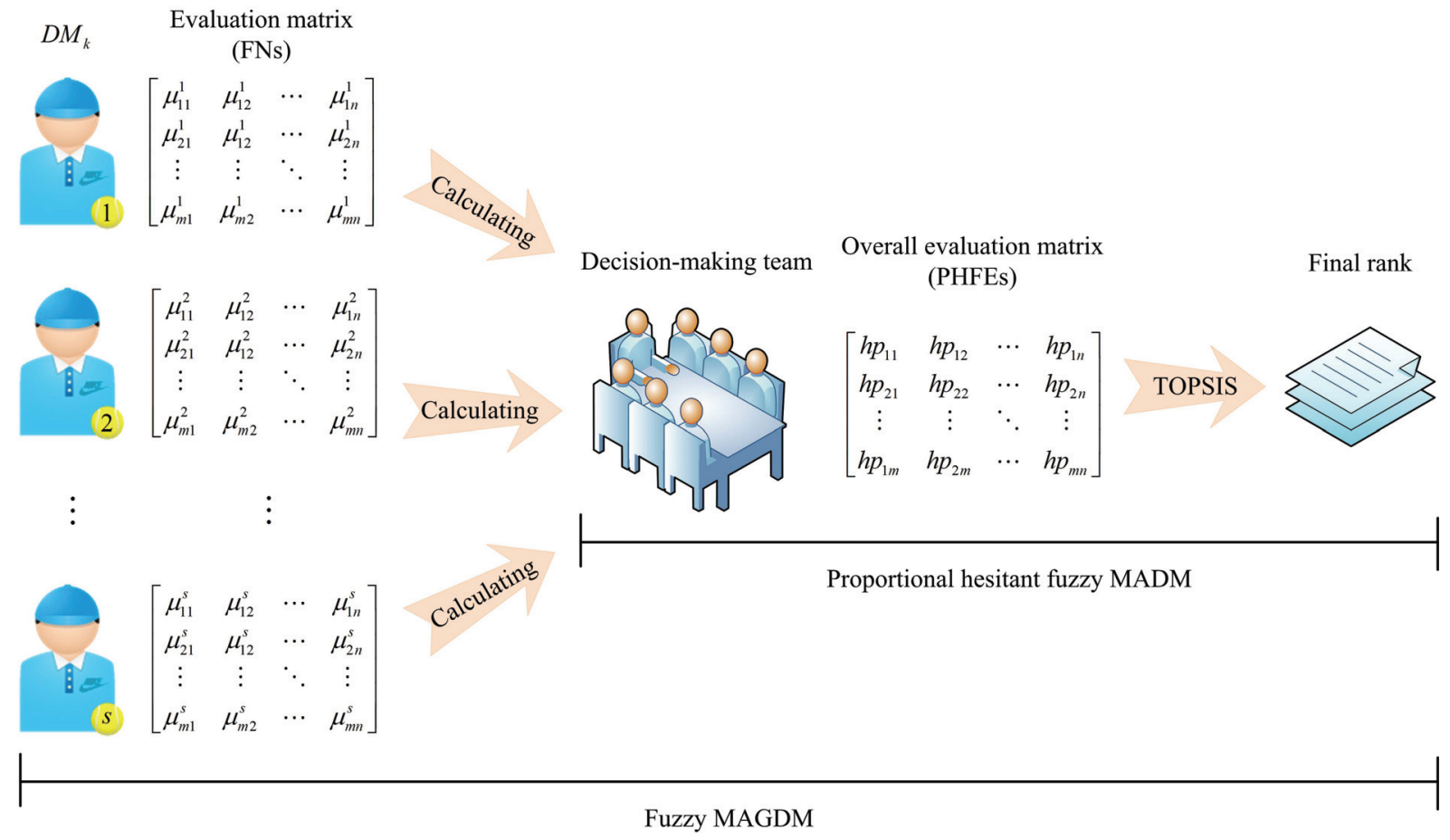

Fig. 1. A novel approach for fuzzy MAGDM.

\subsection{Proportional hesitant fuzzy TOPSIS approach for MAGDM}

Based on the above analysis, the main steps of the proportional hesitant fuzzy TOPSIS approach for the fuzzy MAGDM are as follows (see Figure 1).

Step 1. Decision makers $D M_{k}(k \in S)$ provide evaluation matrices $U_{k}=\left[\mu_{i j}^{k}\right]_{m \times n}(k \in S)$ with the classical FNs. 
Step 2. Calculate the overall evaluation information (see Example 2). The overall evaluation matrix is represented as $U=\left[p h_{i j}\right]_{m \times n}$, where $p h_{i j}(i \in$ $M, j \in N)$ is a PHFE.

Step 3. Because all elements in the overall evaluation matrix $U$ are expressed with PHFEs, there is no need to normalize them.

Step 4. Determine the positive and negative ideal solutions. Based on Definitions 12 and 13 , the positive ideal solution (PIS) is $U^{+}=$ $\left\{p h_{1}^{+}, p h_{2}^{+}, \ldots, p h_{n}^{+}\right\}$and the negative ideal solution (NIS) is $U^{-}=\left\{p h_{1}^{-}, p h_{2}^{-}, \ldots, p h_{n}^{-}\right\}$, where

$p h_{j}^{+}=\left\{\begin{array}{l}\max _{1 \leqslant i \leqslant m} p h_{i j}, \quad \text { for benefit attribute } C_{j}, j \in N \\ \min _{1 \leqslant i \leqslant m} p h_{i j}, \quad \text { for cost attribute } C_{j}, j \in N\end{array}\right.$

and

$p h_{j}^{-}=\left\{\begin{array}{l}\min _{1 \leqslant i \leqslant m} p h_{i j}, \quad \text { for benefit attribute } C_{j}, j \in N \\ \max _{1 \leqslant i \leqslant m} p h_{i j}, \quad \text { for cost attribute } C_{j}, j \in N\end{array}\right.$

Step 5. Measure the distances from positive and negative ideal solutions. Combining the proportional hesitant normalized Hamming distance, the separations of each alternative from the PIS are given as

$$
S_{i}^{+}=d\left(U^{+}, U_{i}\right), i \in M,
$$

where $U_{i}=\left\{p h_{i 1}, p h_{i 2}, \ldots, p h_{i n}\right\}$.

Similarly, the separations of each alternative from the NIS are given as

$$
S_{i}^{-}=d\left(U^{-}, U_{i}\right), i \in M .
$$

Step 6. Calculate the closeness coefficients to the ideal solutions. The closeness coefficient of alternative $A_{i}$ with respect to the ideal solutions is

$$
\operatorname{Coef}_{i}=\frac{S_{i}^{-}}{S_{i}^{+}+S_{i}^{-}}, i \in M .
$$

Step 7. Rank all alternatives. The larger the Coef $_{i}$, the better the alternative $A_{i}, i \in M$.

The novel fuzzy MAGDM approach proposed in this paper has the following main advantages. First, the preferences of the decision-making team for each alternative under different attributes, measured by the proportions, are considered into the decision-making process to improve the reliability of the assessment result. Utilizing PHFEs, the fuzzy MAGDM can be converted into the proportional hesitant fuzzy MADM, which may reduce the complexity of the decision-making system. Finally, the proposed approach can objectively solve the fuzzy MAGDM problem with having a clear understanding on whether an alternative is good at or bad in some attributes.

\subsection{Numerical example}



Samsung - Galaxy S6 edge+ 4G LTE with 32GB Memory Cell Phone - Black Sapphire (Sprint)

Model: SPHG92832BKS | SKU: 4312100 | Customer Rating: ther 4.7 (469 customer reviews)
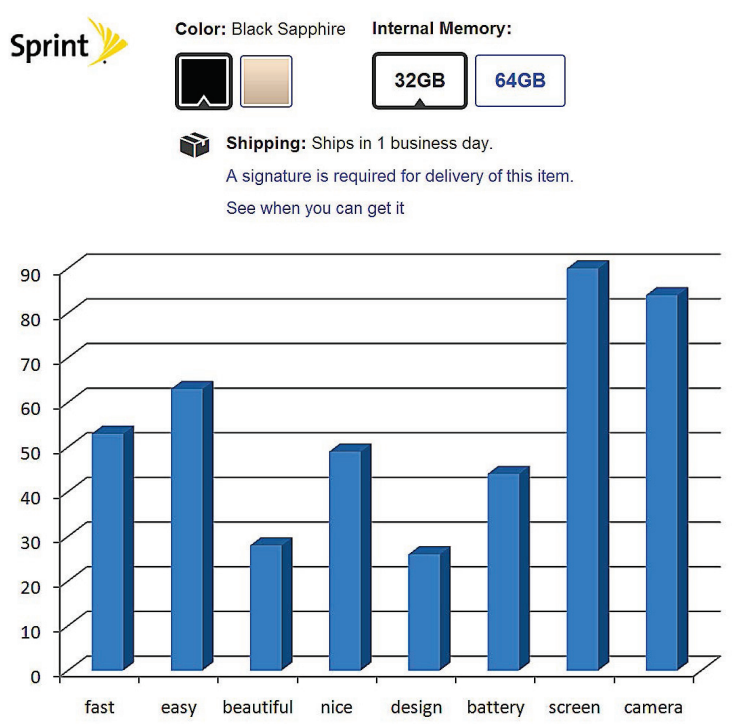

Fig. 2. Customer reviews for a smart-phone.

In practice, in order to evaluate the costperformance of a product, we should first consider how much "performance" it has. Figure 2 shows some keywords and their frequencies of customer reviews for a smart-phone sold in Best Buy. ${ }^{48} \mathrm{Up}$ to March 14, 2016, there are 469 reviews and keyword "screen" appeared 90 times. According to Figure 2, the main factors that involve in the cus- 
tomer reviews and affect the performance of a smartphone can be summarized as follows: $C_{1}$ : system optimization, $C_{2}$ : appearance and system UI design, and $C_{3}$ : hardware configuration. Consider a problem that a decision-making team consisted of five decision makers $D M_{k}(k=1,2, \ldots, 5)$ is invited to evaluate the performance of four smart-phones $A_{i}(i=1,2, \ldots, 4)$. Using FNs, five evaluation matrices are provided as follows.

$$
\begin{aligned}
U_{1} & =\left[\begin{array}{lll}
0.82 & 0.67 & 0.73 \\
0.65 & 0.44 & 0.53 \\
0.13 & 0.42 & 0.14 \\
0.17 & 0.15 & 0.63
\end{array}\right], \\
U_{2} & =\left[\begin{array}{lll}
0.17 & 0.22 & 0.73 \\
0.65 & 0.44 & 0.18 \\
0.81 & 0.36 & 0.14 \\
0.32 & 0.32 & 0.36
\end{array}\right], \\
U_{3} & =\left[\begin{array}{lll}
0.17 & 0.26 & 0.73 \\
0.65 & 0.44 & 0.98 \\
0.81 & 0.36 & 0.14 \\
0.17 & 0.15 & 0.63
\end{array}\right], \\
U_{4} & =\left[\begin{array}{lll}
0.82 & 0.53 & 0.73 \\
0.35 & 0.54 & 0.23 \\
0.69 & 0.36 & 0.14 \\
0.37 & 0.32 & 0.36
\end{array}\right], \\
U_{5} & =\left[\begin{array}{lll}
0.12 & 0.65 & 0.24 \\
0.35 & 0.44 & 0.76 \\
0.81 & 0.36 & 0.14 \\
0.32 & 0.46 & 0.63
\end{array}\right] .
\end{aligned}
$$

To solve this problem, we conduct the MAGDM approach proposed in Subsection 4.1 as follows:

Step 1. Based on the evaluation matrices $U_{k}(k=$ $1,2, \ldots, 5)$, the overall evaluation matrix is $U=$ $\left[p h_{i j}\right]_{4 \times 3}$, where

$$
\begin{aligned}
& p h_{11}=\{(0.82,0.40),(0.17,0.40),(0.12,0.20)\}, \\
& p h_{12}=\{(0.67,0.20),(0.65,0.20),(0.53,0.20), \\
&(0.26,0.20),(0.22,0.20)\}, \\
& p h_{13}=\{(0.73,0.80),(0.24,0.20)\}, p h_{21}=\{(0.65,0.60),(0.35,0.40)\}, \\
& p h_{22}=\{(0.54,0.20),(0.44,0.80)\}, \\
& p h_{23}=\{(0.98,0.20),(0.76,0.20),(0.53,0.20), \\
&(0.23,0.20),(0.18,0.20)\}, \\
& p h_{31}=\{(0.81,0.60),(0.69,0.20),(0.13,0.20)\}, \\
& p h_{32}=\{(0.42,0.20),(0.36,0.80)\}, \\
& p h_{33}=\{(0.14,1.00)\},
\end{aligned}
$$

$$
\begin{aligned}
& p h_{41}=\{(0.37,0.20),(0.32,0.40),(0.17,0.40)\}, \\
& p h_{42}=\{(0.46,0.20),(0.32,0.40),(0.15,0.40)\}, \\
& p h_{43}=\{(0.63,0.60),(0.36,0.40)\} . \\
& \text { Step 2. Following Definition 12, the values of the }
\end{aligned}
$$
score and deviation functions for the elements in the overall evaluation matrix $U$ are shown in Table 2.

Step 3. Because all attributes are benefit attributes, based on Definition 13, the positive ideal solution is

$$
U^{+}=\left\{p h_{31}, p h_{12}, p h_{13}\right\},
$$

and the negative ideal solution is

$$
U^{-}=\left\{p h_{41}, p h_{42}, p h_{33}\right\} .
$$

Step 4. Based on Definition 11, the separations of each alternative from the PIS are $S_{1}^{+}=0.0350, S_{2}^{+}=$ $0.1465, S_{3}^{+}=0.1277, S_{4}^{+}=0.1394$, and the separations of each alternative from the NIS are $S_{1}^{-}=$ $0.1433, S_{2}^{-}=0.1941, S_{3}^{-}=0.1051, S_{4}^{-}=0.0985$.

Step 5. The closeness coefficients of each alternative with respect to the ideal solutions are $\operatorname{Coef}_{1}=$ 0.8037, Coef $_{2}=0.5698$, Coef $_{3}=0.4514$, Coef $_{4}=$ 0.4141 .

Step 6. The ranking order of all smart-phones on the performance is $A_{1} \succ A_{2} \succ A_{3} \succ A_{4}$.

Therefore, Smart-phone $A_{1}$ possesses the best performance. This is because $A_{1}$ not only contains a relatively perfect appearance and system UI design, but also has the best hardware configuration (see Table 1). Although the producer of $A_{3}$ is not good at the appearance and system UI design, he does the best job in the system optimization with the worst hardware configuration. Consequently, the manufacturer of $A_{1}$ may consider cooperating with the producer of $A_{3}$ on the system optimization.

Under the system optimization $\left(C_{1}\right), p h_{41}=$ $\{(0.37,0.20),(0.32,0.40),(0.17,0.40)\} \quad$ indicates that all decision makers think the evaluation value for Smart-phone $A_{4}$ is no more than 0.37 , and $p h_{31}=\{(0.81,0.60),(0.69,0.20),(0.13,0.20)\}$ represents that $60 \%$ decision makers think that for Smart-phone $A_{3}$ is 0.81 . Therefore, $A_{3}$ is better than $A_{4}$ under attribute $C_{1}$. Because the producer of $A_{3}$ does the best job in this attribute, PHFE $p h_{31}$ then is the positive ideal value under the system optimization. Similarly, PHFEs $p h_{12}$ and $p h_{13}$ are the positive ideal values with respect to attributes $C_{2}$ 
Table 2. Values of score and deviation functions for elements in matrix $U$

\begin{tabular}{llllllllll}
\hline & \multicolumn{3}{c}{$C_{1}$} & \multicolumn{3}{c}{$C_{2}$} & \multicolumn{3}{c}{$C_{3}$} \\
\cline { 2 - 10 } & Score & Deviation & Rank & Score & Deviation & Rank & Score & Deviation & Rank \\
\hline$A_{1}$ & 0.420 & 0.107 & 3 & 0.466 & 0.037 & 1 & 0.632 & 0.038 & 1 \\
$A_{2}$ & 0.530 & 0.022 & 2 & 0.460 & 0.002 & 2 & 0.536 & 0.094 & 2 \\
$A_{3}$ & 0.650 & 0.070 & 1 & 0.372 & 0.001 & 3 & 0.140 & 0.000 & 4 \\
$A_{4}$ & 0.270 & 0.007 & 4 & 0.280 & 0.014 & 4 & 0.522 & 0.017 & 3 \\
\hline
\end{tabular}

and $C_{3}$, which is the key factor that contributes to $A_{1}$ having the shortest distance from the PIS.

According to the concept of TOPSIS, the higher rank indicates that an alternative is closer to PIS and farther from NIS simultaneously. Smart-phone $A_{2}$ appears better than $A_{3}$ and $A_{4}$ because of the farthest distance from the NIS (i.e., $S_{2}^{-}=\max _{i=1,2,3,4}\left\{S_{i}^{-}\right\}$), however, the farthest distance from the PIS (i.e., $S_{2}^{+}=\max _{i=1,2,3,4}\left\{S_{i}^{+}\right\}$) brings it a lower rank than Smart-phone $A_{1}$. Besides, doing the worst job in the system optimization and appearance and system UI design leads Smart-phone $A_{4}$ to the last rank.

\section{Comparison}

The proposed PHFSs incorporates the proportional information into HFSs, this section is devoted to clarifying the necessity of our conceptual extension in this paper by providing a comparison between the proportional hesitant fuzzy TOPSIS approach and the hesitant fuzzy TOPSIS approach.

\subsection{Hesitant fuzzy TOPSIS approach for MAGDM}

Ignoring the proportional information, the main steps of the hesitant fuzzy TOPSIS approach for the MAGDM are:

Step $1^{\prime}$. Decision makers $D M_{k}(k \in S)$ provide evaluation matrices $U_{k}=\left[\mu_{i j}^{k}\right]_{m \times n}(k \in S)$ with the classical FNs.

Step $2^{\prime}$. Calculate the overall evaluation information. The overall evaluation matrix is represented as $U^{\prime}=\left[h_{i j}\right]_{m \times n}$, where $h_{i j}(i \in M, j \in N)$ is a HFE.
The fuzzy MAGDM is therefore converted into the hesitant fuzzy MADM, which can be solved by utilizing the hesitant fuzzy TOPSIS approach proposed by $\mathrm{Xu}$ and $\mathrm{Zhang}{ }^{37}$, however, we use the hesitant normalized Hamming distance to measure the distance between HFEs.

Similar to the proportional hesitant fuzzy TOPSIS approach proposed in Subsection 4.1, the hesitant fuzzy TOPSIS approach can objectively solve the fuzzy MAGDM problem with having a clear understanding on whether an alternative is good at or bad in some attributes. Transforming the fuzzy MAGDM into the hesitant fuzzy MADM may also reduce the complexity of the decision-making system. However, the proportional information (or the preferences of the decision-making team) is not considered into the decision process.

\subsection{Dealing with numerical example in Subsection 4.2 through hesitant fuzzy TOPSIS approach}

Based on hesitant fuzzy TOPSIS approach, the numerical example in Subsection 4.2 can be similarly solved as follows:

Step $1^{\prime}$. According to the evaluation matrices $U_{k}(k=1,2, \ldots, 5)$, the overall evaluation matrix is $U^{\prime}=\left[h_{i j}\right]_{4 \times 3}$, where

$$
\begin{aligned}
& h_{11}=\{0.82,0.17,0.12\}, \\
& h_{12}=\{0.67,0.65,0.53,0.26,0.22\}, \\
& h_{13}=\{0.73,0.24\}, \\
& h_{21}=\{0.65,0.35\}, \\
& h_{22}=\{0.54,0.44\}, \\
& h_{23}=\{0.98,0.76,0.53,0.23,0.18\}, \\
& h_{31}=\{0.81,0.69,0.13\}, \\
& h_{32}=\{0.42,0.36\},
\end{aligned}
$$




$$
\begin{aligned}
& h_{33}=\{0.14\}, \\
& h_{41}=\{0.37,0.32,0.17\}, \\
& h_{42}=\{0.46,0.32,0.15\}, \\
& h_{43}=\{0.63,0.36\} .
\end{aligned}
$$

Step $2^{\prime}$. Following Definition 3, the score function values for the elements in the overall evaluation matrix $U^{\prime}$ are shown in Table 3.

Table 3. Values of score function for elements in $U^{\prime}$

\begin{tabular}{lllllll}
\hline & \multicolumn{2}{c}{$C_{1}$} & \multicolumn{2}{c}{$C_{2}$} & \multicolumn{2}{c}{$C_{3}$} \\
\cline { 2 - 7 } & Score & Rank & Score & Rank & Score & Rank \\
\hline$A_{1}$ & 0.370 & 3 & 0.466 & 2 & 0.485 & 3 \\
$A_{2}$ & 0.500 & 2 & 0.490 & 1 & 0.536 & 1 \\
$A_{3}$ & 0.543 & 1 & 0.390 & 3 & 0.140 & 4 \\
$A_{4}$ & 0.287 & 4 & 0.310 & 4 & 0.495 & 2 \\
\hline
\end{tabular}

Step $3^{\prime}$. Based on Definition 4, the positive ideal solution is $U^{\prime+}=\left\{h_{31}, h_{22}, h_{23}\right\}$, and the negative ideal solution is $U^{\prime-}=\left\{h_{41}, h_{42}, h_{33}\right\}$.

Step $4^{\prime}$. Suppose the decision makers are all pessimistic. Following Definition 5, the separations of each alternative from the PIS are $S_{1}^{\prime+}=0.1907$, $S_{2}^{+}=0.0800, S_{3}^{\prime+}=0.1653, S_{4}^{\prime+}=0.2309$, and the separations of each alternative from the NIS are $S_{1}^{\prime-}=0.2606, S_{2}^{\prime-}=0.2409, S_{3}^{\prime-}=0.1267,{S_{4}^{\prime}}^{-}=$ 0.1183 .

Step $5^{\prime}$. The closeness coefficients of each alternative with respect to the ideal solutions are $\operatorname{Coef}_{1}^{\prime}=$ 0.5774, Coef $_{2}^{\prime}=0.7507$, Coef $_{3}^{\prime}=0.4338$, Coef $_{4}^{\prime}=$ 0.3388 .

Step $6^{\prime}$. The ranking order using the hesitant fuzzy TOPSIS approach then is $A_{2} \succ A_{1} \succ A_{3} \succ A_{4}$.

\subsection{Discussion}

The ranking order of all alternatives obtained by the hesitant fuzzy TOPSIS approach is $A_{2} \succ A_{1} \succ A_{3} \succ$ $A_{4}$, whereas it is $A_{1} \succ A_{2} \succ A_{3} \succ A_{4}$ gained by the proportional hesitant fuzzy TOPSIS approach proposed in Subsection 4.1. The difference is the ranking order between $A_{1}$ and $A_{2}$, i.e., $A_{2} \succ A_{1}$ for the former while $A_{1} \succ A_{2}$ for the latter. The main reason is that the proportional hesitant fuzzy TOPSIS approach considers both the membership degrees and their associated proportions into the decision pro- cess, whereas the hesitant fuzzy TOPSIS approach only focuses on the membership degrees but ignores the proportional information. Comparing with the latter, the proportional hesitant fuzzy TOPSIS approach has the following advantages:

(1) Ignoring the proportions may lead to inaccurate average evaluation values for the hesitant fuzzy information. For example, $p h_{13}=$ $\{(0.73,0.80),(0.24,0.20)\}$ indicates that most of the decision makers provide a relatively good evaluation for alternative $A_{1}$ under attribute $C_{3}$. Then, the average evaluation value of it should be more than $0.73 \times 0.8=0.584$, which is larger than $s_{\mathrm{HFE}}\left(h_{23}\right)=$ $s\left(p h_{23}\right)=0.536$. However, under attribute $C_{3}$, the average evaluation value of $A_{1}$ is less than that of $A_{2}$ by utilizing the hesitant fuzzy TOPSIS approach (see Table 2). Therefore, considering the proportional information in the proportional hesitant fuzzy TOPSIS approach may improve the rationality of the positive/negative ideal solution.

(2) In terms of the distance measure, as mentioned in Subsection 3.1, the processes (i.e., "ordering" and "adding") without changing the average evaluation value in each PHFE are beneficial to obtain a relatively accurate distance measure. Therefore, the proportional hesitant fuzzy TOPSIS approach may contribute to more accurate separations of each alternative from the PIS/NIS.

(3) For the proportional information ignored in the hesitant fuzzy TOPSIS approach, the proportional hesitant fuzzy TOPSIS approach regards it as the preferences of the decision-making team, which may increase the reliability of the decision result.

\section{Conclusions}

In this paper, in view of past studies cannot reasonably model a practical case in which a team could not reach agreement on a fuzzy decision, and the proportions of the membership degrees are measurable, the concept of PHFSs has been proposed. As the component of PHFSs, PHFEs contain two aspects of information: the possible membership values and their associated proportions. Because the proportions represent the preferences of the decision-making team, the PHFSs appear more 
accurate and reasonable than HFSs to model the uncertainty produced by human being's doubt. According to Rodríguez et al., ${ }^{4}$ the main advantages of PHFSs and their operations are summarized as follows.

(1) Following the Fundamental Principle of a Generalization $^{4}$, the PHFS is not only a mathematic extension of the HFS, but also a more accurate representation of people's hesitancy in stating their preferences over objects. The novel representation has a large number of applications in practice.

(2) The repeated values in decision making problem are usually removed within HFSs, whereas PHFSs convert them into the proportions, which also may decrease the degree of the hesitancy.

(3) In terms of the distance measure on PHFSs, the ordering method on the basis of both the membership degree and its associated proportion may contribute to reasonable orders of the elements in PHFEs. For the adding method, any addition does not change the distance measure value between two PHFEs.

Besides, a novel MAGDM approach for the fuzzy information has also been developed in this paper. First, the fuzzy MAGDM (expressed by classical FNs) is converted into the proportional hesitant fuzzy MADM (expressed by PHFEs) by calculating the proportions of the associated membership degrees. After that, the alternatives are ranked by the proportional hesitant fuzzy TOPSIS approach. This proposal is different from the traditional fuzzy MAGDM approach, in which the evaluation values are always represented by the FNs as shown in Stage 1 of Section 4. A numerical example is provided to illustrate the fuzzy multiple attribute group decision making process.

As future work, we consider the study of the related operations and properties on PHFSs according to HFSs. Especially, the union and intersection operations on PHFEs in this paper are defined from the angle of probability with the assumption that all PHFEs are mutually independent (see Definition 9). Therefore, defining such operations without that assumption or on the basis of the t-norms and $\mathrm{t}$-conorms ${ }^{49}$ could be a fruitful research of our work.

\section{Acknowledgments}

This work was supported by the Theme-based Research Projects of the Research Grants Council (Grant no. T32-101/15-R) and partly supported by the Key Project of the National Natural Science Foundation of China (Grant No. 71231007)the National Natural Science Foundation of China (Grant No. 71373222) and the CAAC Scientific Research Base on Aviation Flight Technology and Safety (Grant No. F2015KF01).

\section{References}

1. V. Torra, Hesitant fuzzy sets, Int. J. Intell. Syst. 25 (6) (2010) 529-539.

2. K. T. Atanassov, Intuitionistic fuzzy sets, Fuzzy Sets Syst. 20 (1) (1986) 87-96.

3. M. Mizumoto and K. Tanaka, Some properties of fuzzy sets of type 2, Inform. Control 31 (4) (1976) 312-340.

4. R. M. Rodríguez, B. Bedregal, H. Bustince, et al., A position and perspective analysis of hesitant fuzzy sets on information fusion in decision making. Towards high quality progress. Inf. Fusion 29 (2016) 89-97.

5. V. Torra and Y. Narukawa, On hesitant fuzzy sets and decision, in: The 18th IEEE Int. Conf. on Fuzzy Syst., (Jeju Island, Korea, 2009), pp. 1378-1382.

6. L. A. Zadeh, Fuzzy sets, Inf. Control 8 (65) (1965) 338-353.

7. R. R. Yager, On the theory of bags, Int. J. General Syst. 13 (1) (1986) 23-37.

8. Z. Q. Liu and S. Miyamoto (eds.), Soft computing and human-centered machines. (Springer, Berlin 2000).

9. M. M. Xia and Z. S. Xu, Hesitant fuzzy information aggregation in decision making, Int. J. Approx. Reason. 52 (3) (2011) 395-407.

10. Z. M. Zhang, Hesitant fuzzy power aggregation operators and their application to multiple attribute group decision making, Inf. Sci. 234 (10) (2013) 150-181.

11. M. M. Xia, Z. S. Xu and N. Chen, Some hesitant fuzzy aggregation operators with their application in group decision making, Group Decis. Negot. 22 (2) (2013) 259-279.

12. B. Zhu, Z. S. Xu and M. M. Xia, Hesitant fuzzy geometric Bonferroni means, Inf. Sci. 205 (1) (2012) 7285.

13. H. C. Liao, Z. S. Xu and M. M. Xia, Multiplicative consistency of hesitant fuzzy preference relation and its application in group decision making, Int. J. Inform. Technol. Decis. Making 13 (1) (2014) 47-76.

14. Z. M. Mu, S. Z. Zeng and T. Balez̈entis, A novel 
aggregation principle for hesitant fuzzy elements, Knowl. Based Syst. 84 (2015) 134-143.

15. W. Zhou and Z. S. Xu, Optimal discrete fitting aggregation approach with hesitant fuzzy information, Knowl. Based Syst. 78 (1) (2015) 22-33.

16. Z. S. Xu and M. M. Xia, Distance and similarity measures for hesitant fuzzy sets, Inf. Sci. 181 (11) (2011) 2128-2138.

17. B. Farhadinia, Distance and similarity measures for higher order hesitant fuzzy sets, Knowl. Based Syst. 55 (2014) 43-48.

18. N. Chen, Z. S. Xu, M. M. Xia, Correlation coefficients of hesitant fuzzy sets and their application to clustering analysis, Appl. Math. Modell. 37 (4) (2013) 21972211.

19. H. C. Liao, Z. S. Xu, X. J. Zeng, Novel correlation coefficients between hesitant fuzzy sets and their application in decision making, Knowl. Based Syst. 82 (2015) 115-127.

20. Z. S. Xu and M. M. Xia, Hesitant fuzzy entropy and cross-entropy and their use in multi-attribute decisionmaking, Int. J. Intell. Syst. 27 (9) (2012) 799-822.

21. B. Farhadinia, Information measures for hesitant fuzzy sets and interval-valued hesitant fuzzy sets, Inf. Sci. 240 (10) (2013) 129-144.

22. R. M. Rodríguez, L. Martínez and F. Herrera, Hesitant fuzzy linguistic terms sets for decision making, IEEE Trans. Fuzzy Syst. 20 (1) (2012) 109-119.

23. C. P. Wei and H. C. Liao, A multigranularity linguistic group decision-making method based on hesitant 2-tuple sets, Int. J. Intell. Syst. 31 (6) (2015) 612-634.

24. S. H. Xiong, Z. S. Chen, Y. L. Li, et al., On extending power-geometric operators to interval-valued hesitant fuzzy sets and their applications to group decision making, Int. J. Inform. Technol. Decis. Making 15 (05) (2016) 1055-1114.

25. B. Zhu, Z. S. Xu, M. M. Xia, Dual hesitant fuzzy sets, J. Appl. Math. 2012 (11) (2012) 1-13.

26. X. L. Zhang and Z. S. Xu, Hesitant fuzzy agglomerative hierarchical clustering algorithms, Int. J. Syst. Sci. 46 (3) (2015) 562-576.

27. J. J. Peng, J. Q. Wang, J. Wang, et al., An extension of ELECTRE to multi-criteria decision-making problems with multi-hesitant fuzzy sets, Inf. Sci. $\mathbf{3 0 7}$ (2015) 113-126.

28. Y. C. Dong, Y. F. Xu, H. Y. Li, On consistency measures of linguistic preference relations, Eur. J. Oper. Res. 189 (2) (2008) 430-444.

29. R. M. Rodríguez, A Labella, L. Martínez, An overview on fuzzy modelling of complex linguistic preferences in decision making, Int. J. Comput. Intell. Syst. 9 (Supp 1) (2016) 81-94.

30. J. H. Wang and J. Hao, A new version of 2-tuple fuzzy linguistic representation model for computing with words, IEEE Trans. Fuzzy Syst. 14 (3) (2006)
435-445.

31. G. Q. Zhang, Y. C. Dong, Y. F. Xu, Consistency and consensus measures for linguistic preference relations based on distribution assessments, Inf. Fusion 17 (1) (2014) 46-55.

32. Z. Wu and J. Xu, Possibility distribution-based approach for MAGDM with hesitant fuzzy linguistic information, IEEE Trans. Cybernetics 46 (3) (2016) 694-705.

33. Y. C. Dong, Y. Z. Wu, H. J. Zhang, et al., Multigranular unbalanced linguistic distribution assessments with interval symbolic proportions, Knowl. Based Syst. 82 (2015) 139-151.

34. Z. Zhang, C. Guo, L. Martínez, Group decision making based on multi-granular distribution linguistic assessments and power aggregation operators, (2015) arXiv: $1504.01004 \mathrm{v} 1$

35. Z. S. Chen, K. S. Chin, Y. L. Li, et al., Proportional hesitant fuzzy linguistic term set for multiple criteria group decision making, Inf. Sci. 357 (2016) 61-87.

36. C. L. Hwang and K. Yoon (eds.), Multiple attributes decision making methods and applications. (Springer, Berlin Heidelberg 1981).

37. Z. S. Xu and X Zhang, Hesitant fuzzy multi-attribute decision making based on TOPSIS with incomplete weight information. Knowl. Based Syst. (2013) $5253-$ 64.

38. I. Beg and T. Rashid, TOPSIS for hesitant fuzzy linguistic term sets, Int. J. Intell. Syst. 28 (12) (2013) 1162-1171.

39. R. M. Rodríguez, L. Martínez, V. Torra, et al. Hesitant fuzzy sets: State of the art and future directions, Int. J. Intell. Syst. 29 (6) (2014) 495-524.

40. B. Farhadinia, Hesitant fuzzy set lexicographical ordering and its application to multi-attribute decision making, Inf. Sci. 327 (2016) 233-245.

41. B. Farhadinia, A series of score functions for hesitant fuzzy sets, Inf. Sci. 277 (2) (2014) 102-110.

42. D. F. Li, Multiattribute decision making models and methods using intuitionistic fuzzy sets, J. Comput. Syst. Sci. 70 (1) (2005) 73-85.

43. B. Zhu and Z. S. Xu, Analytic hierarchy processhesitant group decision making, Eur. J. Oper. Res. 239 (3) (2014) 794-801.

44. R. Pérez-Fernández, P. Alonso, H. Bustince, et al., Ordering finitely generated sets and finite interval-valued hesitant fuzzy sets, Inf. Sci. 325 (2015) 375-392.

45. R. Pérez-Fernández, P. Alonso, H. Bustince, et al., Applications of finite interval-valued hesitant fuzzy preference relations in group decision making, Inf. Sci. 326 (2016) 89-101.

46. Y. C. Dong, H. Zhang, E. Herrera-Viedma, Consensus reaching model in the complex and dynamic MAGDM problem, Knowl. Based Syst. (2016) 106 206-219. 
47. Y. C. Dong, Y. Liu, H. Liang, et al., Strategic weight manipulation in multiple attribute decision making, Omega (2017) IDO:doi.org/10.1016/j.omega.2017.02.008

48. Best Buy, Samsung - Galaxy S6 edge+ 4G LTE with 32GB Memory Cell Phone - Black Sapphire (Sprint), (2016) http://www.bestbuy.com/site/samsu ng-galaxy-s6-edge-4g-lte-with-32gb-mem ory-cell-phone-black-sapphire-sprint/ 4312100 . p? $i d=1219729639321 \&$ skuId $=4312100$ (accessed 14-March-16).

49. Z. S. Chen, K. S. Chin, Y. L. Li, et al. On generalized extended Bonferroni means for decision making. IEEE Trans. Fuzzy Syst. (2016) 24 (6) 1525-1543. 